\title{
Modeling the X-ray emission of SN 1993J
}

\author{
T. K. Nymark ${ }^{1}$, P. Chandra ${ }^{2,3}$, and C. Fransson ${ }^{1}$ \\ 1 Department of Astronomy, Stockholm University, AlbaNova University Center, 10691 Stockholm, Sweden \\ e-mail: tanja@astro.su.se \\ 2 Jansky Fellow, National Radio Astronomy Observatory, USA \\ 3 Department of Astronomy, University of Virginia, PO Box 400325, Charlottesville, VA 22904-4325, USA
}

Received 31 August 2008 / Accepted 28 November 2008

\section{ABSTRACT}

\begin{abstract}
Aims. We investigate the effects of radiative shocks on the observed X-ray emission from the type II supernova SN 1993J. To this end, the X-ray emission is modeled as a result of the interaction between the supernova ejecta and a dense circumstellar medium at an age of 8 years.

Methods. The circumstances under which the reverse shock is radiative are discussed and the observed X-ray emission is analyzed using the numerical code described in Nymark et al. (2006, A\&A, 449, 171). We argue that the original analysis of the X-ray observations suffered from the lack of self-consistent models for cooling shocks with high density and velocity, leading to questionable conclusions about the temperatures and elemental abundances. We reanalyze the spectra with our numerical model, and discuss the expected spectra for different explosion models for the progenitors.

Results. We find that the spectra of SN 1993J are compatible with a CNO-enriched composition and that the X-ray flux is dominated by the reverse shock.
\end{abstract}

Key words. supernovae: individual: SN 1993J - stars: circumstellar matter - X-rays: stars - hydrodynamics - shock waves atomic processes

\section{Introduction}

Many core-collapse supernovae (SNe) show strong X-ray and radio emission. This is, in particular, true for the type IIL, IIn and IIb SNe (see e.g. Immler \& Lewin 2003, for a review). We now know that this is a result of the interaction of the supernova ejecta and the circumstellar medium (CSM) of the supernova. A twoshock structure is formed consisting of the outgoing supernova shock (hereafter the forward shock) and a reverse shock, which moves backward into the ejecta (Chevalier 1982a,b; Chevalier \& Fransson 1994, hereafter CF94). The region behind the forward shock consists of shocked circumstellar material, with $T \sim 10^{8}-10^{9} \mathrm{~K}$, while the region behind the reverse shock, which consists of shocked ejecta, is cooler, with $T \sim 10^{7} \mathrm{~K}$. The radio emission is caused by synchrotron emission from the forward shock (e.g. Sramek \& Weiler 2003), while the X-ray emission originates partly from free-free and inverse Compton emission behind the forward shock, and partly from line emission in the cooler gas behind the reverse shock. Both the radio and the X-ray emission are powerful tools for understanding the physics of both the SNe and the CSM.

With the new generation of X-ray telescopes, XMM-Newton and Chandra, more detailed spectra have been obtained than ever before. This puts greater demands on the tools used for analyzing the spectra. Traditionally, X-ray spectra have been analyzed with the XSPEC package, which includes a number of models that can be combined to get the best fit. In Nymark et al. (2006, hereafter NFK06) we showed, however, that the SNe having the strongest $\mathrm{X}$-ray emission are likely to have radiative shocks. This has not been accounted for in the analysis of the SNe observed in $\mathrm{X}$-rays. Furthermore, none of the models included in the XSPEC distribution offers the possibility of fitting cooling shocks in a consistent manner.

In radiative shocks a wide range of temperatures contribute to the spectrum. The cooling also affects the hydrodynamic structure and influences the emitting volume, and thus the total luminosity from the interaction region. This is not properly accounted for when fitting with one, or even several, singletemperature models, and the elemental abundances can be overor under-estimated in the resulting analysis. In NFK06 we described a numerical model that computes the emission from a cooling shock self-consistently. This was applied to the coronal emission from the ring collision in SN 1987A by Gröningsson et al. (2006). Here we apply this model to the X-ray spectra of SN 1993J, obtained with Chandra (Swartz et al. 2003) and XMM-Newton (Zimmermann \& Aschenbach 2003).

\section{Observations and data analysis}

SN 1993J was discovered on March 28, 1993 in M 82 (Ripero \& Garcia 1993). It was one of the brightest supernovae of the twentieth century, and has been followed in great detail in many wavelengths in the decade following the explosion. On the basis of the detection of hydrogen lines in the spectrum SN 1993J was first identified as a type II supernova, but as the Balmer lines soon weakened its spectrum came to resemble that of a type Ib supernova, and it was re-classified as a type IIb (Filippenko et al. 1993). This transition is explained by an almost complete 
loss of the hydrogen envelope due to heavy pre-supernova mass loss, probably due to a binary interaction (e.g. Nomoto et al. 1993; Podsiadlowski et al. 1993; Ray et al. 1993; Woosley et al. 1994). This hypothesis was confirmed by Maund et al. (2004), when they detected the unambiguous signature of a massive star, i.e., the binary companion of the supernova progenitor, in HST observations of the supernova 10 years after the explosion. Evidence of circumstellar interaction was seen at early times in radio (Van Dyk et al. 1994), UV (Fransson \& Sonneborn 1994), X-rays (Zimmerman et al. 1994; Uno et al. 2002) and $\gamma$-rays by OSSE on CGRO (Leising et al. 1994). The detection of the supernova in the radio (Chandra et al. 2004; Weiler et al. 2007) and X-rays bands (Uno et al. 2002) even after a decade shows the presence of an extended dense CSM.

During the first weeks ROSAT and ASCA observations showed a hard X-ray spectrum. This was confirmed by OSSE, which showed a hard spectrum, well fit by a free-free spectrum with $k T \sim 100 \mathrm{keV}$ (Leising et al. 1994). However, after six months ASCA observations showed that this had changed to a soft spectrum (Uno et al. 2002). This transition is consistent with circumstellar interaction, as the decreasing optical depth in the expanding cool shell allows more of the soft emission to escape (Fransson et al. 1996, hereafter FLC96).

In May 2000 SN 1993J was observed with Chandra (Swartz et al. 2003). Strong Fe L emission is evident in the spectrum, as are emission lines from Mg XI-XII and Si XIII-XIV. Swartz et al. (2003) fit the spectrum with three components - two low temperature components $(k T \sim 0.35 \mathrm{keV}$ and $k T \sim 1.01 \mathrm{keV})$ with no $\mathrm{He}, \mathrm{C}, \mathrm{O}$ and $\mathrm{Ne}$ but enhanced in $\mathrm{N}, \mathrm{Mg}$, Si and Fe; as well as a high temperature component $(k T \sim 6.0 \mathrm{keV})$, consistent with solar abundances (Swartz et al. 2003). This was argued to be consistent with an interaction model, in which the high temperature component comes from the circumstellar shock, while the two low-temperature components come from different parts of a radiative reverse shock.

In April 2001 Zimmermann \& Aschenbach (2003) observed SN 1993J with XMM-Newton. Their spectrum was found to be best fit with two components (with $k T \sim 0.34 \mathrm{keV}$ and $k T \sim 6.4 \mathrm{keV})$. These are in good agreement with the high and low temperature components of the Chandra spectrum, but the intermediate component found by Swartz et al. (2003) in the Chandra spectrum is missing in the analysis of the XMM spectrum by Zimmermann \& Aschenbach (2003). This could be caused by a real change in the spectrum between the two observations. Also here the two components are taken as evidence of a two-shock structure, but they found the abundances to be poorly constrained, and no conclusions were drawn about the composition of the ejecta.

As we argue below, both these analyses are doubtful if the shock is radiative, as it most likely is. The abundances were kept as completely free parameters, and it is not clear how unique the solution is, especially with a realistic temperature model. The thin hydrogen envelope means that the reverse shock will traverse the hydrogen rich region on a short timescale, and the emission will already after a year be dominated by processed material, which gives us an excellent opportunity for testing stellar evolution theories. In this paper we take a different approach and use abundance models based on self-consistent stellar evolution models as input.

For the analysis in this paper we make new extractions of both the Chandra and XMM-Newton data to fit with our models. Here we describe the observational and data analysis procedure.

\subsection{The Chandra dataset}

The parent galaxy of SN 1993J, M 81, was observed with a 50 ks exposure on May 7, 2000 with the Chandra Advanced CCD Imaging Spectrometer (ACIS) spectroscopy array operating in imaging mode (Swartz et al. 2003). We refer to Swartz et al. (2003) for the technical details of the observations. We extracted the data from the ChandraXO archive and analyzed this using standard CIAO analysis threads and XSPEC (Arnaud 1996). In order to check for any X-ray flaring events, we selected a background region of size 90", carefully excluding point sources. We used a radius of $7 "$ centered at the SN 1993J position to extract the SN events; and an annulus of inner radius 7" and outer radius $18^{\prime \prime}$ centered at the supernova position to obtain the background counts. We refer to Swartz et al. (2003) and Chandra et al. (2005) for the data analysis procedure. We binned the data in $15 \mathrm{cts}$ per channel for the spectral analysis. The observed spectrum is shown in Fig. 1. While analysing the supernova spectrum with XSPEC, we ignored the counts below $0.2 \mathrm{keV}$ and above $6.0 \mathrm{keV}$, since there was almost no flux in these energy ranges.

\subsection{The $X M M$ dataset}

XMM-Newton observed SN 1993J on April 22/23, 2001, for a total of $132 \mathrm{ks}$. The $90 \mathrm{ks}$ data was with the PN camera in the small window mode and $83 \mathrm{ks}$ with the MOS2 camera in image mode. The medium filter was applied both in the PN and the MOS2 instrument setup during the observation (Zimmermann \& Aschenbach 2003). Due to the presence of the variable particle background in parts of the observing period, we flagged the flaring events from the light curve. In the PN observations about half of the observations were affected by bursts in the particle background. We extracted the spectrum and created response matrices using XMM-SAS. The spectrum was binned as group of 50 bins. The observed spectrum is shown in Fig. 1. Spectral fits were performed using only the PN camera data offering the best statistics.

\section{Modeling}

In general, the X-ray flux is a combination of the flux from the forward and reverse shocks. As we will show below, in most cases the reverse shock is likely to be radiative. For these cases we have made a self-consistent model in which hydrodynamic calculations for a stationary flow are combined with timedependent ionization balance and multi-level atoms, including optical depth effects (NFK06). This means that the emission in the cooling flow behind the reverse shock is followed from the shock to the cool shell behind the shock, giving correct weight to the emission from each zone. The parameters of the shock calculations are the reverse shock temperature, the density and the chemical composition, and the output is the emitted spectrum. For the cases where the cooling time is long we assume a one-temperature spectrum.

Ionization rates are from Arnaud \& Rothenflug (1985) and Arnaud \& Raymond (1992), while recombination rates are from Nahar (1996, 1999), Nahar \& Pradhan (1997), and Zatsarinny et al. (2003, 2004a,b). Most of the collisional and radiative data are from the Chianti database, version 4.2 (Dere et al. 1997; Young et al. 2003) ${ }^{1}$. For further details we refer to NFK06.

1 CHIANTI is a collaborative project involving the NRL (USA), RAL (UK), and the following universities: College London (UK), Cambridge (UK), George Mason (USA), and Florence (Italy). 

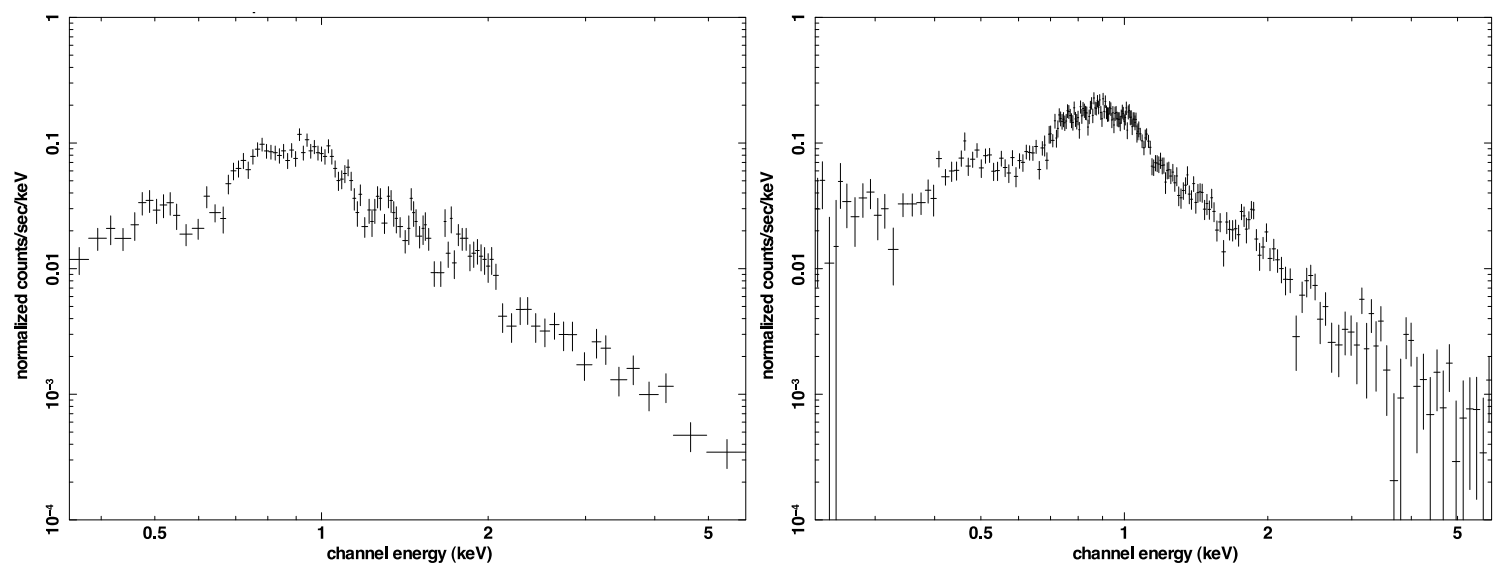

Fig. 1. The Chandra dataset (left) at $t=2594$ days since explosion and the XMM dataset (right) at $t=2943$ days since explosion.

\section{Structure of the ejecta}

The properties of the circumstellar interaction depend on the density structure in the ejecta. Explosion models for red supergiants indicate that the density distribution in the ejecta can be described by a power law, $\rho \propto v^{-\eta}$. For a polytrope of index $n=3 / 2$, typical of red supergiants, Matzner \& McKee (1999) have shown that $\eta \approx 10$ in the outer layers. Deviations from a simple polytropic progenitor may, however, result in a more complicated structure. As an example, the model 4H47, specifically calculated for SN 1993J (Shigeyama et al. 1994; Nomoto \& Hashimoto 1988), shows a structure with a steep outer part and a flatter inner profile, and then an additional steepening further in, as well as considerable structure in the inner parts (see their Fig. 3). This profile is, however, also a simplification, since Iwamoto et al. (1997) find that especially the inner, steep region is Rayleigh-Taylor unstable.

As the reverse shock moves inward in the ejecta, it passes slowly through the steep outer gradient, while moving more rapidly in the flatter part before eventually reaching the second steep gradient. When the gradient changes, the spectrum is also expected to change as the reverse shock slows down (steep part) or speeds up (flat part). There is evidence that this has happened in SN 1993J (Dwarkadas et al. 2005).

The shock radius depends on time as $R_{\mathrm{S}} \propto t^{m}$, where the expansion parameter $m=(\eta-3) /(\eta-2)$. Numerical calculations by Dwarkadas et al. (2005) based on the $4 \mathrm{H} 47$ model indicate that a change in the expansion parameter should occur at approximately day 2300 . Such a change was indeed observed by Bartel et al. (2002) in the radio at $\sim 1400$ days. For the cases discussed in the present work it is worth noting the difference in the X-ray spectra from Chandra (Swartz et al. 2003) on day 2594 and the one from XMM (Zimmermann \& Aschenbach 2003) a year later. Although both these observations were done somewhat later than the expected change in the spectrum, the observed difference could be related to a change in the expansion parameter.

Models of the light curve and spectrum of SN 1993J strongly indicate that less than $0.5 M_{\odot}$ of the hydrogen envelope was left at the time of the explosion (e.g. Woosley et al. 1994; Nomoto et al. 1993; Ray et al. 1993; Houck \& Fransson 1996). Further, explosion models like the $4 \mathrm{H} 47$ model by Nomoto \& Hashimoto (1988) and Shigeyama et al. (1994) predict that the outer parts of the ejecta should contain nearly equal mass fractions of $\mathrm{H}$ and $\mathrm{He}$ as a result of $\mathrm{CNO}$ processing.
Assuming a constant density gradient in the ejecta and a steady wind, we can estimate the swept up mass and therefore what the composition at the reverse shock should be. If the density of the CSM is given by

$\rho_{\mathrm{w}}=\frac{\dot{M}}{4 \pi R_{\mathrm{s}}^{2} v_{\mathrm{w}}}$,

the mass swept up by the reverse shock is

$M_{\mathrm{rev}}=\frac{\eta-4}{2} \frac{\dot{M}}{v_{\mathrm{w}}} R_{\mathrm{s}}$

where $v_{\mathrm{w}}$ is the wind velocity (Chevalier 1982b). With $R_{\mathrm{s}}=V_{\mathrm{ej}} t$, we get

$$
\begin{aligned}
M_{\mathrm{rev}}= & 1.3 \times 10^{-2}(\eta-4)\left(\frac{V_{\mathrm{ej}}}{10^{4} \mathrm{~km} \mathrm{~s}^{-1}}\right) \\
& \times\left(\frac{\tilde{A_{*}}}{4}\right)\left(\frac{t_{\mathrm{d}}}{1000 \text { days }}\right) M_{\odot}
\end{aligned}
$$

where $V_{\mathrm{ej}}$ is the ejecta velocity and $t_{\mathrm{d}}$ the time since explosion in days. For SN $1993 \mathrm{~J} \tilde{A_{*}} \equiv\left(\dot{M} / 10^{-5} M_{\odot} \mathrm{yr}^{-1}\right) /\left(v_{\mathrm{w}} / 10 \mathrm{~km} \mathrm{~s}^{-1}\right) \approx 4$ (FLC96). For the Chandra observations we take $t_{\mathrm{d}}=2600$ and $V_{4}=V_{\mathrm{ej}} / 10^{4} \mathrm{~km} \mathrm{~s}^{-1} \approx 1$, which is consistent with the expansion velocity derived from VLBI observations at this time (Bartel et al. 2002, 2007).

Assuming an ejecta density gradient $\eta=25$ in the outer zones, and $\eta=5$ in the shallow middle zone, as indicated by the 4H47 model by Shigeyama et al. (1994), we find the swept up mass at 2600 days to be $\sim 0.38 M_{\odot}$. The hydrogen-rich envelope in the $4 \mathrm{H} 47$ model has a mass of $0.47 M_{\odot}$. Inside this envelope the outer part of the He zone is N-rich, while the inner part is C-rich. The $\mathrm{He} / \mathrm{N}$ zone contains $0.3 M_{\odot}$, and the $\mathrm{He} / \mathrm{C}$ zone contains $1.5 M_{\odot}$.

The estimate above shows that the reverse shock may be close to the border between the H-rich envelope and the He-rich zone. However, this estimate depends on the mass assumed for the hydrogen envelope. With a lower hydrogen mass, as in the s15s7b model by Woosley et al. (1994), where the hydrogen envelope is only $0.2 M_{\odot}$, it is therefore quite possible that the reverse shock is somewhere in the He-rich zone. Nevertheless, we cannot exclude the possibility that clumps of metal-rich material may have reached the outer parts by mixing, as indicated by the simulations by Iwamoto et al. (1997). In Sect. 8 we therefore also discuss the spectrum resulting from metal-rich compositions. 


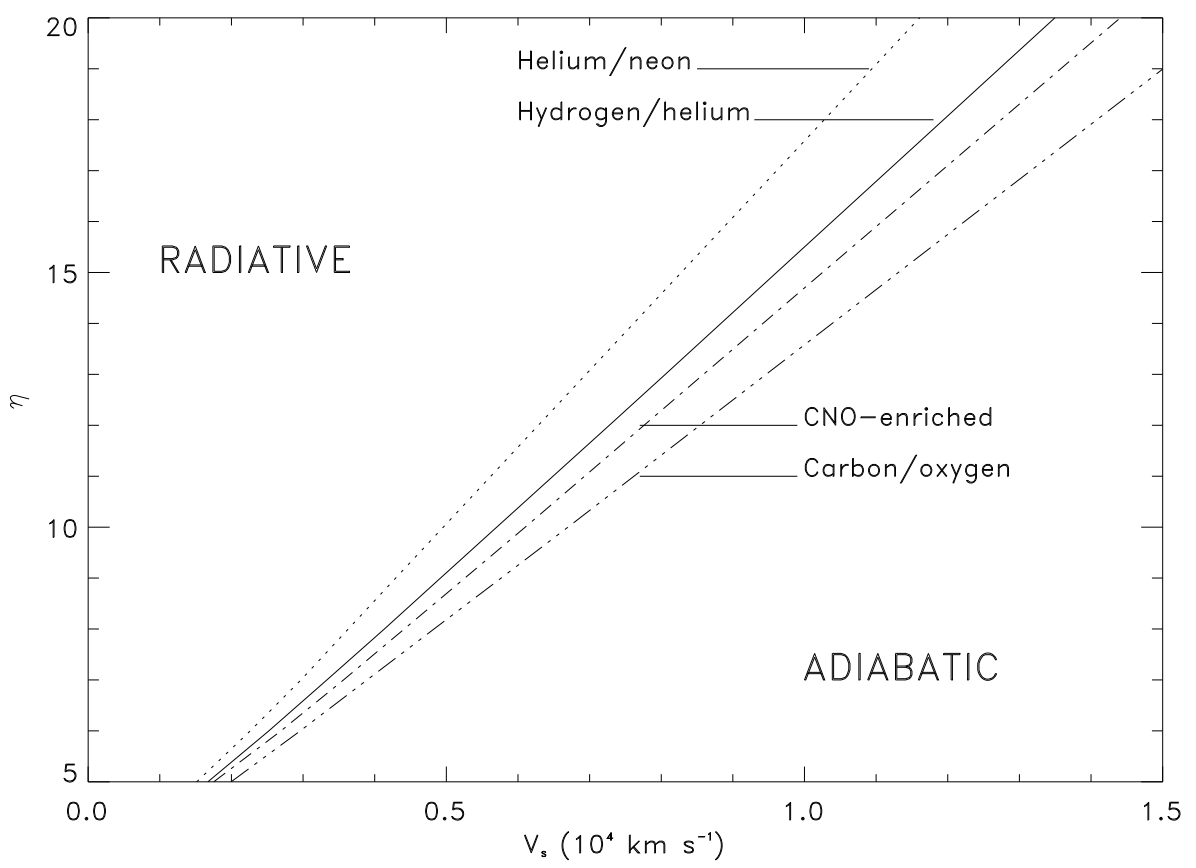

Fig. 2. The lines represent the border between the radiative and adiabatic regimes for each composition. In each case the region above the line corresponds to the reverse shock being radiative, while values of $\eta$ and $V_{\mathrm{s}}$ below the line lead to adiabatic shocks.
Swartz et al. (2003) found that the Chandra spectrum of SN 1993J was best fit with a composition dominated by $\mathrm{H}$, and where $\mathrm{N}, \mathrm{Mg}, \mathrm{Si}$, and $\mathrm{Fe}$ were enhanced with respect to solar abundances, while $\mathrm{He}, \mathrm{C}, \mathrm{O}$ and $\mathrm{Ne}$ were absent. These abundances are, however, not consistent with the calculated models for the ejecta. In the analysis by Zimmermann \& Aschenbach (2003) the XMM observations were fit with a number of different models, all with widely differing elemental abundances, but these abundances were very poorly constrained.

\section{Radiative shocks}

The structure of the reverse shock was discussed in NFK06, where we showed that this can remain radiative for several years, and longer. The emission and cooling depend on the reverse shock temperature, the pre-shock density and the composition. These are the fundamental parameters, but each of these are related to the shock velocity, $V_{\mathrm{s}}$, mass loss parameter, $A_{*}$, ejecta density gradient, $\eta$, and the time since explosion, $t$, by the relations discussed in NFK06. For clarity we here repeat the relevant relations.

An estimate of whether the shock is radiative can be found by comparing the expansion time with the cooling timescale. In NFK06 we showed that the ratio between these timescales can be written as

$$
\frac{t_{\text {cool }}}{t}=\frac{6.7 \times 10^{-22} \mu_{\mathrm{A}}^{2} \mu V_{4}^{4} t_{\mathrm{d}}}{\left(\mu_{\mathrm{A}}-\mu\right)(\eta-2)^{2}(\eta-3)(\eta-4) \Lambda\left(T_{\mathrm{rev}}\right) \tilde{A}_{*}},
$$

where $\mu$ is the mean mass per particle and $\mu_{\mathrm{A}}$ is the mean atomic weight. The cooling function, $\Lambda\left(T_{\text {rev }}\right)$ is discussed in NFK06. The shock is radiative if $t_{\text {cool }} \lesssim t$. If the interaction region is thin compared with the size of the supernova, as will be the case if the shock is radiative, the thin shell approximation of Chevalier (1982b) applies. In this case the temperature of the reverse shock is given by

$T_{\mathrm{rev}}=\frac{2.27 \times 10^{9} \mu}{(\eta-2)^{2}} V_{4}^{2}$.
Using this in Eq. (4) and requiring that $t_{\text {cool }} / t \lesssim 1.0$ we find that the shock is radiative if the reverse shock temperature fulfills the requirement

$$
\begin{aligned}
& T_{\text {rev }} \lesssim \frac{8.8 \times 10^{19}}{\mu_{\mathrm{A}}}(\eta-2) \\
& \times\left(\mu\left(\mu_{\mathrm{A}}-\mu\right)(\eta-3)(\eta-4) \Lambda(T) \tilde{A}_{*} t_{\mathrm{d}}^{-1}\right)^{\frac{1}{2}} .
\end{aligned}
$$

Table 2 shows the limiting temperatures, $T_{\text {rad }}$, and expected shock temperatures for a number of compositions and density gradients for the case of SN 1993J, with $\tilde{A_{*}}=4$ and $t_{\mathrm{d}}=$ 2600 days. $T_{\text {rad }}$ is defined as the limiting temperature corresponding to $t_{\text {cool }}=t$, such that if $T_{\text {rev }}<T_{\text {rad }}$ the shock will be radiative while $T_{\text {rev }}>T_{\text {rad }}$ gives an adiabatic shock. These regimes are also illustrated in Fig. 2 in terms of the ejecta density gradient and the shock velocity. The lines in Fig. 2 represent the border between the adiabatic and radiative regimes for a few different compositions. If the ejecta density gradient and the shock velocity are in the region above the line corresponding to the relevant composition, the shock is radiative. This means that for a given shock velocity, the shock is more likely to be radiative the higher the density gradient is. We emphasize that the values of $\eta$ and $V_{\mathrm{s}}$ corresponding to radiative and adiabatic shocks shown in Fig. 2 are a function of the time since the explosion, the mass loss rate and the cooling rate, and are therefore only valid for the specific case discussed here.

Swartz et al. (2003) fit the SN 1993J data with two low temperature components $(k T=0.35 \mathrm{keV}$ and $k T=1.0 \mathrm{keV})$ and one high temperature component $(k T=6.0 \mathrm{keV})$. From Table 2 we find that the $k T_{\text {rev }}=0.35 \mathrm{keV}$ shock is likely to be radiative, and, depending on the composition, also the $k T_{\text {rev }}=1.0 \mathrm{keV}$ component, while a $k T_{\text {rev }}=6.0 \mathrm{keV}$ schock is probably adiabatic. This means that the assumption of a single-temperature shock is not realistic for the two low-temperature components, and their analysis is therefore not consistent.

It is possible that the high energy component comes from the hot outer shock, while the $k T_{\text {rev }}=1.0 \mathrm{keV}$ component comes from a radiative reverse shock. In this case, the $k T_{\text {rev }}=0.35 \mathrm{keV}$ component could be representative of the cooling part downstream from the reverse shock. However, as we discuss below, 
Table 1. Fractional abundances by number and molecular weights for the compositions discussed here.

\begin{tabular}{|c|c|c|c|c|c|c|c|c|}
\hline & Solar & $\mathrm{H} / \mathrm{He}$ zone & $\begin{array}{c}4 \mathrm{H} 47 \\
\mathrm{He} / \mathrm{N} \text { zone }\end{array}$ & $\mathrm{C} / \mathrm{O}$ zone & $\mathrm{H} / \mathrm{He}$ zone & $\begin{array}{c}\mathrm{s} 15 \mathrm{~s} 7 \mathrm{~b} \\
\mathrm{He} / \mathrm{N} \text { zone }\end{array}$ & $\mathrm{C} / \mathrm{O}$ zone & SN 1987A-like \\
\hline $\mathrm{H}$ & $9.10 \times 10^{-1}$ & $7.41 \times 10^{-1}$ & - & - & $8.87 \times 10^{-1}$ & - & - & $7.99 \times 10^{-1}$ \\
\hline $\mathrm{He}$ & $8.89 \times 10^{-2}$ & $2.57 \times 10^{-1}$ & $9.95 \times 10^{-1}$ & $9.66 \times 10^{-2}$ & $1.11 \times 10^{-1}$ & $9.95 \times 10^{-1}$ & - & $2.0 \times 10^{-1}$ \\
\hline $\mathrm{C}$ & $3.79 \times 10^{-4}$ & $3.01 \times 10^{-5}$ & $6.77 \times 10^{-5}$ & $4.07 \times 10^{-1}$ & $2.23 \times 10^{-4}$ & $8.76 \times 10^{-5}$ & $2.36 \times 10^{-1}$ & $1.03 \times 10^{-4}$ \\
\hline $\mathrm{N}$ & $7.92 \times 10^{-5}$ & $1.29 \times 10^{-3}$ & $2.92 \times 10^{-3}$ & - & $3.49 \times 10^{-4}$ & $3.64 \times 10^{-3}$ & - & $5.15 \times 10^{-4}$ \\
\hline $\mathrm{O}$ & $6.29 \times 10^{-4}$ & $3.38 \times 10^{-5}$ & $5.08 \times 10^{-5}$ & $4.80 \times 10^{-1}$ & $6.99 \times 10^{-4}$ & $6.49 \times 10^{-5}$ & $7.43 \times 10^{-1}$ & $4.69 \times 10^{-4}$ \\
\hline $\mathrm{Ne}$ & $8.89 \times 10^{-5}$ & $7.86 \times 10^{-4}$ & $1.77 \times 10^{-3}$ & $1.23 \times 10^{-2}$ & $1.19 \times 10^{-4}$ & $3.56 \times 10^{-4}$ & $1.93 \times 10^{-2}$ & $8.89 \times 10^{-5}$ \\
\hline $\mathrm{Mg}$ & $3.62 \times 10^{-5}$ & $3.57 \times 10^{-5}$ & $8.03 \times 10^{-5}$ & $3.21 \times 10^{-3}$ & $3.92 \times 10^{-5}$ & $1.17 \times 10^{-4}$ & $7.69 \times 10^{-4}$ & $3.63 \times 10^{-5}$ \\
\hline $\mathrm{Si}$ & $3.46 \times 10^{-5}$ & $4.42 \times 10^{-5}$ & $9.94 \times 10^{-5}$ & $3.25 \times 10^{-4}$ & $3.73 \times 10^{-5}$ & $1.11 \times 10^{-4}$ & $4.16 \times 10^{-4}$ & $3.45 \times 10^{-5}$ \\
\hline S & $1.69 \times 10^{-5}$ & $2.13 \times 10^{-5}$ & $4.78 \times 10^{-5}$ & $1.56 \times 10^{-4}$ & $1.81 \times 10^{-5}$ & $5.40 \times 10^{-5}$ & $2.02 \times 10^{-4}$ & $1.69 \times 10^{-5}$ \\
\hline $\mathrm{Ar}$ & $3.62 \times 10^{-6}$ & $9.30 \times 10^{-6}$ & $2.08 \times 10^{-5}$ & $6.13 \times 10^{-5}$ & $3.63 \times 10^{-6}$ & $1.08 \times 10^{-5}$ & $4.05 \times 10^{-5}$ & $3.62 \times 10^{-6}$ \\
\hline $\mathrm{Ca}$ & $2.13 \times 10^{-6}$ & $2.63 \times 10^{-6}$ & $5.93 \times 10^{-6}$ & $1.94 \times 10^{-5}$ & $2.23 \times 10^{-6}$ & $6.66 \times 10^{-6}$ & $2.49 \times 10^{-5}$ & $2.13 \times 10^{-6}$ \\
\hline $\mathrm{Fe}$ & $3.08 \times 10^{-5}$ & $5.30 \times 10^{-5}$ & $1.19 \times 10^{-4}$ & $3.90 \times 10^{-4}$ & $3.28 \times 10^{-5}$ & $9.79 \times 10^{-5}$ & $3.66 \times 10^{-4}$ & $3.08 \times 10^{-5}$ \\
\hline $\mathrm{Ni}$ & $1.62 \times 10^{-6}$ & $2.78 \times 10^{-6}$ & $2.22 \times 10^{-6}$ & $2.05 \times 10^{-5}$ & $1.72 \times 10^{-6}$ & $5.14 \times 10^{-6}$ & $1.92 \times 10^{-5}$ & $1.62 \times 10^{-6}$ \\
\hline$\mu_{\mathrm{A}}$ & 1.29 & 1.81 & 4.07 & 11.37 & 1.36 & 4.06 & 15.20 & 1.63 \\
\hline$\mu$ & 0.61 & 0.80 & 1.34 & 1.70 & 0.64 & 1.34 & 1.77 & 0.74 \\
\hline
\end{tabular}

Table 2. Reverse shock temperature, $k T_{\text {rev }}$ and limiting temperature, $k T_{\text {rad }}$ (both in $\mathrm{keV}$ ) for a radiative shock, for SN 1993J at 2600 days for $\dot{M}=4.0 \times 10^{-5} M_{\odot} \mathrm{yr}^{-1}$.

\begin{tabular}{l|cc|cc|cc}
\hline \hline \multicolumn{1}{c|}{$\eta$} & \multicolumn{2}{|c|}{7} & \multicolumn{2}{c|}{12} & \multicolumn{2}{c}{20} \\
Composition & $k T_{\text {rad }}$ & $k T_{\text {rev }}$ & $k T_{\text {rad }}$ & $k T_{\text {rev }}$ & $k T_{\text {rad }}$ & $k T_{\text {rev }}$ \\
\hline Solar & 0.51 & 4.77 & 0.63 & 1.19 & 0.68 & 0.37 \\
H/He (4H47) & 0.69 & 6.30 & 0.82 & 1.57 & 0.88 & 0.48 \\
He/N (4H47) & 0.93 & 10.5 & 1.04 & 2.60 & 1.09 & 0.81 \\
C/O (4H47) & 2.02 & 13.5 & 2.43 & 3.39 & 2.61 & 1.04 \\
H/He (s15s7b2f) & 0.53 & 5.01 & 0.66 & 1.25 & 0.71 & 0.39 \\
He/N (s15s7b2f) & 0.86 & 10.4 & 0.99 & 2.60 & 1.04 & 0.80 \\
C/O (s15s7b2f) & 2.32 & 13.9 & 2.80 & 3.46 & 3.00 & 1.07 \\
SN 1987A-like & 0.73 & 5.70 & 0.87 & 1.40 & 0.92 & 0.44 \\
\hline
\end{tabular}

in Sect. 6, the temperature of the outer shock is unlikely to be as low as $6.0 \mathrm{keV}$. Another possibilty is the presence of several shocks in the ejecta, for example if the ejecta is clumpy. In that case all three components could come from the reverse shock, but corresponding to different angles with respect to the clumpy ejecta. This alternative is discussed in more detail in Sect. 7.

\section{Emission from the circumstellar shock}

At late times the reverse shock is expected to dominate the X-ray emission. There may, however, still be a contribution from the outer shock, in particular at energies $\gtrsim 5 \mathrm{keV}$. The high temperature component found in the fits to the Chandra and XMM observations of SN 1993J was attributed to the circumstellar shock, which is expected to have a considerably higher temperature than the reverse shock. It is surprising, though, that this temperature is as low as $\sim 6.0 \mathrm{keV}$. The shocked CSM has an ion temperature of (FLC96)

$T_{\mathrm{cs}}=2.27 \times 10^{9} \mu \frac{(\eta-3)^{2}}{(\eta-2)^{2}} V_{4}^{2} \mathrm{~K}$,

where $\mu=0.61$ for a solar composition, and given in Table 1 for other compositions. For $V_{4}=1$ and $\eta=12, k T_{\mathrm{cs}} \gtrsim 100 \mathrm{keV}$. Allowing for the possibility of a lower ejecta density gradient or shock velocity, this value may be reduced somewhat, but it is difficult to get a temperature lower than $\sim 40 \mathrm{keV}$ if the ion temperature is equal to the electron temperature.
However, if collisionless heating is inefficient, the electron temperature can be considerably lower than the ion temperature (e.g., FLC96). A lower limit to the electron temperature may be found from the timescale for electron-ion equilibration

$$
\begin{aligned}
t_{\mathrm{e}-\mathrm{i}} & \approx 29\left(\frac{T_{\mathrm{e}}}{10^{9} \mathrm{~K}}\right)^{3 / 2}\left(\frac{n_{\mathrm{e}}}{10^{8} \mathrm{~cm}^{-3}}\right)^{-1} \\
& \approx 0.21\left(\frac{T_{\mathrm{e}}}{10^{9} \mathrm{~K}}\right)^{3 / 2}{\tilde{A_{*}}}^{-1} V_{4}^{2} t_{\mathrm{d}}^{2} \text { days. }
\end{aligned}
$$

Collisionless heating by plasma instabilities may, however, increase the electron temperature. There is, however, evidence from observations of galactic supernova remnants that collisionless heating is only effective for low Mach number shocks (Ghavamian et al. 2007; Vink et al. 2003). Because of the much higher densities at these comparatively early stages, it is, however, not clear how these results apply to our cases.

Equating $t_{\mathrm{e}-\mathrm{i}}$ to the expansion time gives

$k T_{\mathrm{e}} \gtrsim 7.4 V_{4}^{-4 / 3}\left(\frac{\tilde{A_{*}}}{4}\right)^{2 / 3}\left(\frac{t_{\mathrm{d}}}{1000 \text { days }}\right)^{-2 / 3} \mathrm{keV}$.

An electron temperature as low as $6.0 \mathrm{keV}$, as found by Swartz et al. (2003), is therefore in principle possible. Also cosmic ray dominated shocks can give a lower temperature and higher compression rate (e.g., Decourchelle et al. 2000; Ellison et al. 2004), which could contribute to lowering the temperature of the forward shock. For efficient cosmic ray acceleration, with an injection efficiency (fraction of protons with superthermal energies) of $\eta=10^{-2}$ at the outer shock the models of Decourchelle et al. (2000) give temperatures of a few $\mathrm{keV}$, while the resulting high compression can lead to relative thickness of the outer shock (as defined below) of $f \approx 0.04$. Also this effect can therefore give a shock temperature of $6.0 \mathrm{keV}$.

However, a second requirement is that this component should also produce the observed luminosity of $1.6 \times$ $10^{38} \mathrm{erg} \mathrm{s}^{-1}$ in the range $2.4-8.0 \mathrm{keV}$. The luminosity from the circumstellar shock is given by

$$
\begin{aligned}
L_{\lambda, \mathrm{cs}} \approx & 2.54 \times 10^{37} \frac{\mu_{\mathrm{A}}-\mu}{\mu \mu_{\mathrm{A}}} \tilde{A}_{*}^{2} V_{4}^{-1}\left(\frac{f}{0.2}\right)^{-1} \\
& \times T_{8}^{-0.236} \lambda^{-2} \mathrm{e}^{-1.44 / \lambda T_{8}} \\
& \times\left(\frac{t_{\mathrm{d}}}{1000 \text { days }}\right)^{-1} \operatorname{erg~s}^{-1} \AA^{-1}
\end{aligned}
$$


using a Gaunt factor $g_{\mathrm{ff}} \sim 1.87 T_{8}^{-0.264}$ (FLC96). In this expression $f$ is the relative thickness of the adiabatic outer shock, defined by $f=\left(R_{\mathrm{cs}}-R_{\mathrm{cd}}\right) / R_{\mathrm{cd}}$, where $R_{\mathrm{cs}}$ is the outer shock radius and $R_{\mathrm{cd}}$ is the radius of the contact discontinuity. For $\eta=7-20$, $f \approx 0.2-0.3$ (CF94). Inserting the appropriate parameters, we find that the expected luminosity in the range $2.4-8.0 \mathrm{keV}$ from a circumstellar component with $k T \sim 6.0 \mathrm{keV}$ is $L_{\mathrm{cs}} \sim 2.0 \times$ $10^{37} \mathrm{erg} \mathrm{s}^{-1}$ at 2600 days, i.e., only about one tenth of the observed luminosity. A higher compression, as resulting from cosmic ray acceleration, can lead to higher luminosity. However, even the most efficient model in Decourchelle et al. (2000), with $\eta=10^{-2}$, which gives a compression of $f \approx 0.04$, will for a temperature of $6.0 \mathrm{keV}$ lead to an increase of the luminosity by only a factor of five, which is not enough to explain the observed luminosity. We conclude that it is unlikely that the circumstellar shock is responsible for the $6.0 \mathrm{keV}$ component. This is in agreement with FLC96 who find that the luminosity of the forward shock after $t \sim 1000$ days is orders of magnitude lower than that of the reverse shock.

\section{Oblique shocks}

If either the CSM or the ejecta is clumpy, parts of the reverse shock will hit these clumps obliquely. The transmitted shocks will then have a lower velocity than the part of the reverse shock which hits the clumps head-on, and also the shock temperature will be lower. This scenario could result in a considerable range of shock velocities contributing to the emission. In particular, even if the head-on shock is adiabatic for the parameters chosen (i.e., a given reverse shock velocity and ejecta density), the tangential shocks may be radiative.

This is similar to the situation found for the ring collision in SN 1987A, where Zhekov et al. (2006) find that a range of shock velocities are necessary to explain the observed spectrum. The presence of both hard X-rays and optical emission from the shocked CSM clearly shows the importance of geometric and complex dynamics of a clumpy CSM. The hydrodynamical simulations by Iwamoto et al. (1997), as well as Kifonidis et al. (2006), however, show that also the ejecta is subject to Rayleigh-Taylor instabilities in connection to the passage of the shock. The same situation as in the CSM case with a range of velocities for the reverse shock will then arise. With this background, we therefore attempt to fit the spectra with two or more shocks, possibly with a mix of adiabatic and radiative shocks. The results of this fitting are discussed in the next section.

\section{Model fitting of the X-ray spectra}

In this section we discuss the modeling of the spectra along the lines of the previous discussion. First we emphasize that we do not aim at perfect statistical fits, keeping all parameters, like temperature, abundances and absorbing column density, free. Instead, our aim is to investigate how well - or how badly the observations are reproduced by different realistic explosion models. Therefore we focus on the important spectral features, and investigate how well each of them is reproduced by each model. In this way we hope to distinguish between different models for the composition, and therefore the nature of the progenitor. We believe this to be more physically illuminating than the more commonly used method of varying all parameters until a best fit has been achieved. In particular, with data of low spectral resolution and moderate $\mathrm{S} / \mathrm{N}$ this can easily result in doubtful abundances. Different sets of abundances may result in similar values of $\chi^{2}$, and the one with the lowest value may have little significance compared to others with substantially different abundances. Since we do not allow all parameters to vary freely, the $\chi^{2}$ statistic is not really appropriate. One approach which is used in similar circumstances is to investigate quantities which can be determined unambiguously in the data and the model (e.g., Badenes et al. 2008). In the present case, however, the quality of the data does not allow any of this to be determined unambiguously. Therefore we choose instead to focus on specific spectral features, and use the $\chi^{2}$ statistic as a guideline to distinguish between a few specific models. This is similar to the approach taken by Rakowski et al. (2006).

We have chosen to relate our models to the ejecta composition found from two explosion models for SN 1993J, the 4H47 model (Nomoto \& Hashimoto 1988; Shigeyama et al. 1994) and the s15s7b model (Woosley et al. 1994). From each of these we have selected a number of compositions, corresponding to the different burning zones in the ejecta (Table 1).

We also discuss a CNO-enriched composition resembling the one in the circumstellar ring of SN 1987A, but with initially three times higher metallicity, to account for the low metallicity of the LMC compared to the more normal metallicity in M 81 and NGC 3877. This composition has been determined in the following way: we took $\mathrm{H} / \mathrm{He}=0.25$ by number, as appropriate for the circumstellar ring of SN 1987A. The sum of C, N and $\mathrm{O}$ abundances was taken to be equal to the solar value, while the ratios were the same as in the ring of SN 1987A, i.e., N/C $=5$ and $\mathrm{N} / \mathrm{O}=1.1$ (Lundqvist \& Fransson 1996). For all other elements we use solar abundances. We note that the optical observations of SN 1993J indicate a high N/C ratio (Fransson et al. 2005).

We also note that due to the presence of the cooling shell, part of the flux density from the reverse shock will be absorbed (e.g. CF94). This absorption will come in addition to the Galactic absorption column density. However, unlike the Galactic absorption, which approximately should have solar abundances, this extra absorption should normally have the abundances of the burning zone close to the shock. We therefore first determine the column density for a nearby source in the field of view of the $\mathrm{SN}$, and freeze the Galactic column density to that value in our fitting. The extra absorption contribution will then solely come from the cooling shock.

For each composition we attempt to fit the spectrum with one reverse shock temperature, which we allow to vary between the maximum reverse shock temperatures corresponding to ejecta density gradients in the range $\eta=7$ to 20 . The corresponding reverse shock temperature, $T_{\text {rev }}$, for each composition is given in Table 2, together with the limiting temperature for a radiative shock, $T_{\text {rad }}$. If the best fit reverse shock temperature exceeds $T_{\text {rad }}$, we take an adiabatic component with a temperature that is allowed to vary between $T_{\text {rad }}$ and $T_{\text {rev }}$.

We also tested adding a hot, single-temperature component with solar abundances, representing the outer shock. For this component we used the XSPEC model "Vmekal", which gives the spectrum from a hot, diffuse gas with a single temperature. This is basically a free-free spectrum for $k T_{\mathrm{cs}} \gtrsim 10 \mathrm{keV}$.

\section{Results}

The XMM-Newton spectrum of SN 1993J (Fig. 1, right hand panel) is dominated by the Fe L emission at $0.7-1.0 \mathrm{keV}$, which is blended with strong Ne IX-X emission. A lower peak is evident at $0.4-0.5 \mathrm{keV}$, most likely caused by strong N VII lines. Several emission lines are present above $\sim 1.0 \mathrm{keV}$, in particular from 
Table 3. Fitting parameters for different components for the SN 1993J XMM-Newton data.

\begin{tabular}{|c|c|c|c|c|c|}
\hline \multirow{2}{*}{\multicolumn{2}{|c|}{ Parameters }} & \multicolumn{4}{|c|}{ Models } \\
\hline & & Solar & $\begin{array}{l}\text { HHe-Zone } \\
(\mathrm{s} 15 \mathrm{~s} 7)\end{array}$ & $\begin{array}{l}\text { HeN-Zone } \\
\text { (s15s7) }\end{array}$ & SN 87A-like \\
\hline \multicolumn{2}{|l|}{$\chi^{2} /$ d.o.f. } & 2.13 & 1.98 & 1.26 & 1.67 \\
\hline \multirow{2}{*}{ Rad. Comp } & Temp (keV) & $0.68 \pm 0.08$ & $0.71 \pm 0.08$ & $1.02 \pm 0.41$ & $0.75 \pm 0.11$ \\
\hline & $\operatorname{Norm}\left(10^{-3}\right)$ & $5.79 \pm 0.11$ & $6.53 \pm 0.83$ & $6.20 \pm 0.23$ & $5.10 \pm 0.73$ \\
\hline \multirow{2}{*}{ Ad. Comp } & Temp (keV) & $1.33 \pm 0.24$ & $1.37 \pm 0.03$ & $2.07 \pm 0.16$ & $1.35 \pm 0.03$ \\
\hline & $\operatorname{Norm}\left(10^{5}\right)$ & $2.08 \pm 0.04$ & $1.81 \pm 0.05$ & $0.28 \pm 0.02$ & $1.58 \pm 0.03$ \\
\hline Unabsorbed flux ${ }^{*}$ & Rad. Comp & $1.12 \pm 0.02$ & $1.24 \pm 0.15$ & $1.64 \pm 0.6$ & $1.14 \pm 0.16$ \\
\hline \multirow{2}{*}{$10^{-13} \mathrm{erg} \mathrm{s}^{-1}$} & Ad. Comp & $1.72 \pm 0.03$ & $1.59 \pm 0.04$ & $1.45 \pm 0.10$ & $1.73 \pm 0.03$ \\
\hline & Total flux & $2.84 \pm 0.05$ & $2.84 \pm 0.19$ & $3.09 \pm 0.16$ & $2.87 \pm 0.19$ \\
\hline \multicolumn{2}{|c|}{$n_{\mathrm{H}}(\mathrm{cool}$ shell $)$ in $10^{20} \mathrm{~cm}^{-2}$} & $13.2 \pm 1.40$ & $11.8 \pm 0.96$ & $0.07 \pm 0.07$ & $6.5 \pm 1.1$ \\
\hline Flux (Reverse Shock) ${ }^{* *}$ & Rad. Comp & $2.49 \pm 0.05$ & $2.46 \pm 0.31$ & $2.73 \pm 0.10$ & $2.18 \pm 0.31$ \\
\hline \multirow[t]{2}{*}{$10^{-13} \mathrm{erg} \mathrm{s}^{-1}$} & Ad. Comp & $2.60 \pm 0.05$ & $2.92 \pm 0.08$ & $1.89 \pm 0.14$ & $2.46 \pm 0.05$ \\
\hline & Total flux & $5.09 \pm 0.10$ & $5.38 \pm 0.39$ & $4.62 \pm 0.24$ & $4.66 \pm 0.36$ \\
\hline
\end{tabular}

* Corrected for the Galactic extinction column density, $n_{\mathrm{H}}=4.17 \times 10^{20} \mathrm{~cm}^{-2}$

** The total flux from the reverse shock before absorption by the cool shell.

Mg XI-XII and Si XIII-XIV, with the Mg XI and Si XIII lines being the stronger, as well as S XVI. The absence of $\mathrm{O}$ emission at $0.6-0.8 \mathrm{keV}$ is striking, as the $\mathrm{O}$ VIII lines at 0.65 and $0.775 \mathrm{keV}$ are expected to be strong over a wide range of temperatures.

The Chandra spectrum (Fig. 1, left hand panel) shows much the same features as the XMM spectrum, but with a more pronounced double appearance of the $\mathrm{Fe} \mathrm{L}$ peak, with one peak at $\sim 0.8 \mathrm{keV}$ and a second peak at $\sim 0.9 \mathrm{keV}$. Because the $\mathrm{S} / \mathrm{N}$ is considerably lower for the Chandra spectrum, we will in the following concentrate on the XMM spectrum.

We will now systematically investigate to what extent the abundance models in Table 1 reproduce these observations. We start with a solar composition, and then go on to more specialized models for different nuclear burning zones.

In the case of SN 1993J Swartz et al. (2003) found the best fit column density for the M 81 nucleus to be $4.4 \times 10^{20} \mathrm{~cm}^{-2}$. Dickey \& Lockman (1990) found that the Galactic absorption column density in that direction is $4.17 \times 10^{20} \mathrm{~cm}^{-2}$, which is well in agreement with that of the best fit column density. Hence we froze the Galactic column density with solar composition to this value.

When fitting the spectrum with one radiative shock, we found that the best fit temperature exceeded $T_{\text {rad }}$ in all models. An adiabatic shock with a higher temperature gave a better fit, but in most cases the best fit was found for a combination of an adiabatic shock and a radiative shock at half the temperature of the adiabatic shock.

Adding a hot component from the circumstellar shock did not improve the fits, and the best fit temperature for this component was $\lesssim 5 \mathrm{keV}$ in all cases. As discussed in Sect. 6, the forward shock is unlikely to have such a low temperature. We therefore conclude that there is no indication of a contribution from the forward shock to the spectrum of SN 1993J. The absorption column density needed is in all cases just a little bit higher than the Galactic column density, which suggests that SN 1993J has significant contribution from the adiabatic reverse shock too, along with the radiative part.

We will now discuss the specific abundance zones one by one. The best fit parameters for the solar composition, the CNOenriched compositions from the model s $15 \mathrm{~s} 7 \mathrm{~b} 2 \mathrm{f}$ (Woosley et al. 1994) and the SN 1987A-like composition are given in Table 3.
The spectra were found to be best fit with one adiabatic component (denoted here by Ad. Comp) and one radiative component (Rad. Comp). For each component the table shows the best fit temperature, and the flux normalization constant (Norm) required to fit the spectrum.

\subsection{A solar composition}

First we note that the relative strengths of the MgXI and Mg XII lines, and of the Si XIII and Si XIV lines, indicate that there is a component with temperature in the range $1.0-2.0 \mathrm{keV}$. This is consistent with a density gradient $\eta \sim 10-12$. A lower temperature gives too strong Mg XI, and Si XIII lines, while a higher temperature gives too strong Mg XII and Si XIV lines. However, temperatures above $1.0 \mathrm{keV}$ strongly overestimate the emission at the Fe $\mathrm{L}$ peak at $0.9 \mathrm{keV}$, while not having sufficient emission below $0.8 \mathrm{keV}$. This may indicate that there is one component responsible for the line emission above $1.0 \mathrm{keV}$, and another, cooler component, responsible for the emission at lower energies.

For a solar composition the $\mathrm{O}$ VIII-emission at $0.6-0.7 \mathrm{keV}$ is prominent. As this is absent in the observed spectrum, a strong absorption is needed to remove this emission. This, however, also removes the $\mathrm{N}$ VII-emission at $0.5 \mathrm{keV}$, which is evident in the spectrum.

We may improve this by adding a second component, which gives a best fit for shocks of $k T_{\text {rev }}=1.0$ and $k T_{\text {rev }}=2.0 \mathrm{keV}$, shown in Fig. 3. We note that while the XMM-observations are reasonably well reproduced in the range $0.8-1.0 \mathrm{keV}$, the Chandra observations show a dip at $0.9 \mathrm{keV}$, which means that this model overestimates the emission there. Both spectra are poorly reproduced below $0.7 \mathrm{keV}$, most likely because of the strong absorption needed to remove the O VIII-emission.

We therefore conclude that a composition with less $\mathrm{O}$ and more $\mathrm{N}$ than the solar composition is needed. This is in agreement with the results from the optical emission, which indicate the presence of CNO-processed material (Fransson et al. 2005). In the following we therefore investigate the spectra produced by compositions taken from the explosion models described above. 

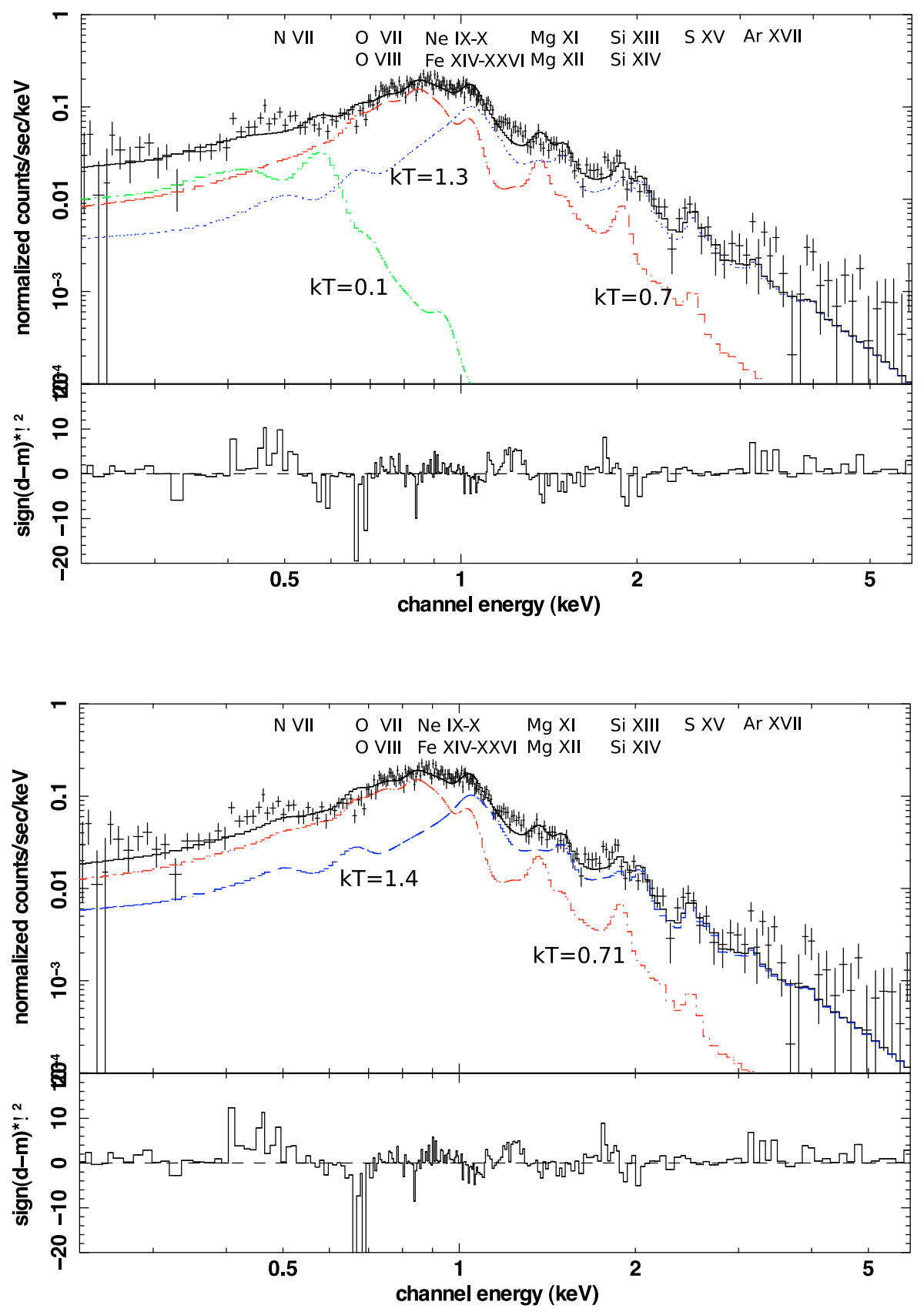

Fig. 3. Fit to the XMM spectrum of SN 1993J for an adiabatic shock with $k T_{\text {rev }}=1.3 \mathrm{keV}$ (dotted curve) and two radiative shock with $k T_{\text {rev }}=0.7 \mathrm{keV}$ (upper dashed curve) and $k T_{\text {rev }}=0.1 \mathrm{keV}$ (lower dashed curve) for a solar composition. The solid curve is the sum of the three components. Note the poor fit at the $\mathrm{N}$ VII peak at $\sim 0.5 \mathrm{keV}$ and the $\mathrm{O}$ VII-VIII emission at $\sim 0.7 \mathrm{keV}$.
Fig. 4. Fit to the spectrum of SN 1993J with an adiabatic shock at $k T_{\text {rev }}=1.4 \mathrm{keV}$ (long dashed) and a radiative shock at $k T_{\text {rev }}=$ $0.71 \mathrm{keV}$ (short dashed) for a composition based on the outer, hydrogen-rich envelope of the $\mathrm{s} 15 \mathrm{~s} 7 \mathrm{~b} 2 \mathrm{f}$ model. Also here the fit to the $\mathrm{N}$ VII and O VII-VIII emission at $0.5-0.7 \mathrm{keV}$ is poor.

\subsection{A CNO enriched hydrogen envelope}

The composition from the outer envelope of the 4H47 model, where the original envelope composition has been modified by $\mathrm{CNO}$-processing in the progenitor (Table 1), gives weaker $\mathrm{O}$-lines than the solar model, while the $\mathrm{N}$-lines are stronger. However, also this composition gives too weak N VII emission at $\sim 0.5 \mathrm{keV}$. These models also show a deficiency at $0.6-0.7 \mathrm{keV}$ compared to the observed spectrum, while the Fe $\mathrm{L} / \mathrm{Ne} \mathrm{X}$ peak at $0.9-1.0 \mathrm{keV}$ is too strong. This indicates that this composition has too much $\mathrm{Ne}$ and too little $\mathrm{O}$. At energies above $\sim 1.0 \mathrm{keV}$ the continuum is too weak, and there is hardly any line emission.

The outer envelope of the s15s7b2f model has more $\mathrm{O}$ and less $\mathrm{Ne}$ than the corresponding zone in the $4 \mathrm{H} 47$ model, giving stronger O VII-VIII lines and a less pronounced Ne X peak. Also, the Fe XVII lines are stronger, as are the Mg XI-XII and
Si XIII-XIV lines at $1.2 \mathrm{keV}$ and $1.8 \mathrm{keV}$. However, although this model does a better job at reproducing the Fe L emission and the line emission above $1.2 \mathrm{keV}$, also in this model the O-emission at $0.7-0.8 \mathrm{keV}$ is underestimated, and the Ne-peak is too strong. In this case two shocks improve the fit, but cannot reproduce the missing O-emission. Figure 4 shows a two-shock model based on the model s1517b2f. In this case the temperatures of the two shocks are 0.7 and $1.4 \mathrm{keV}$.

As a realistic $\mathrm{CNO}$-enriched composition we have also tested the SN 1987A-like composition discussed in Sect. 8. The fit obtained with this composition is better than with the other two CNO-enriched compositions, although the N VII peak at $0.5 \mathrm{keV}$ also in this case is underproduced, while the Fe XVII emission at $0.8 \mathrm{keV}$ is too strong.

Adding a second shock improves the fit further by allowing strong line emission at high energies without overestimating the 

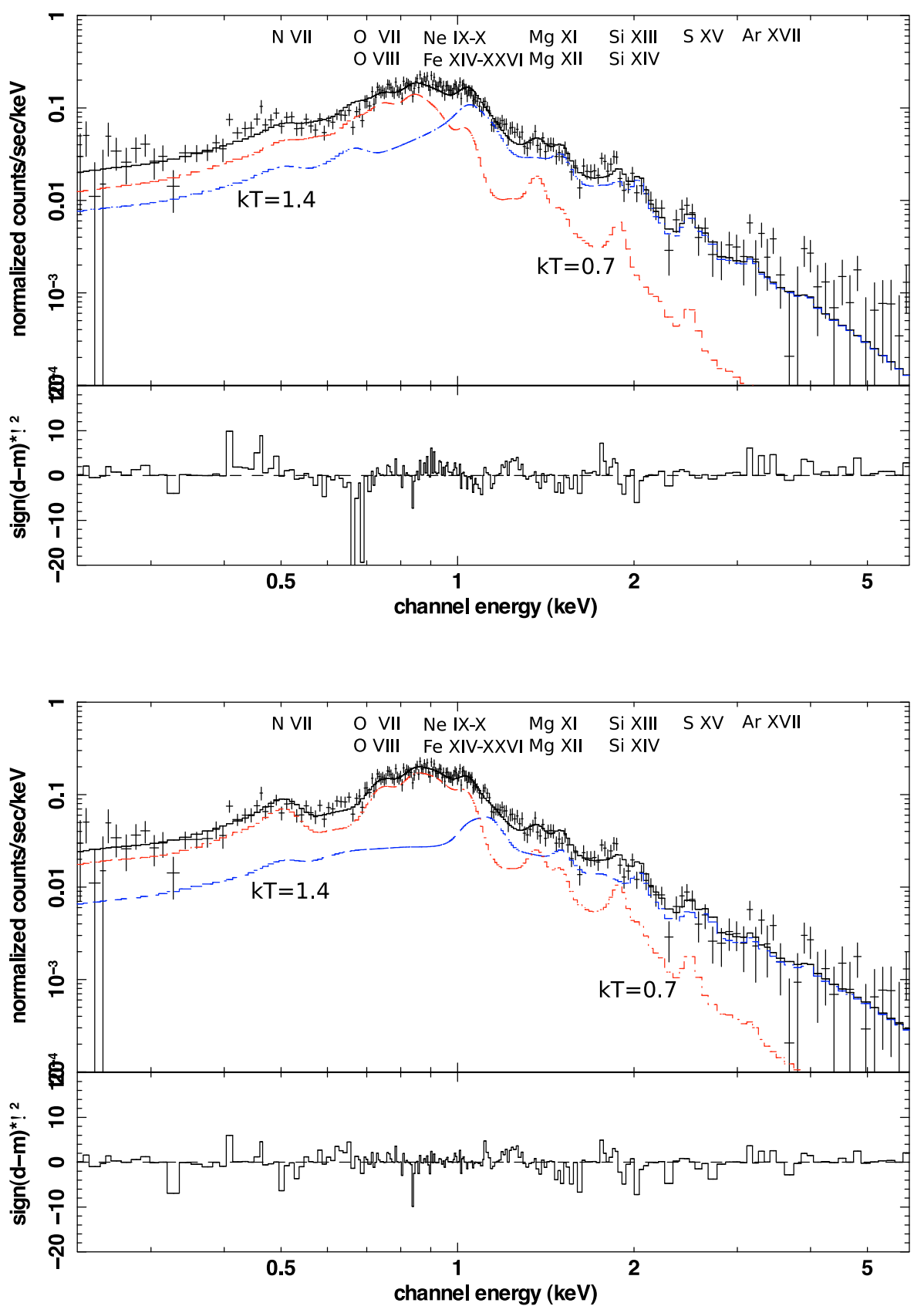

Fig. 5. Fit to the XMM spectrum of SN 1993J with a composition resembling that of the circumstellar ring of SN 1987A, but with enhanced metal abundances. The fit consists of an adiabatic shock at $k T_{\text {rev }}=1.4 \mathrm{keV}$ (dotted) and a radiative shock at $k T_{\text {rev }}=0.7 \mathrm{keV}$ (dashed). The fit to the N VII peak at $\sim 0.5 \mathrm{keV}$ has improved somewhat compared to Figs. 3 and 4, but the $\mathrm{O}$ VII-VIII emission at $0.7 \mathrm{keV}$ is still poorly fit.
Fig. 6. Fit to the XMM spectrum of SN 1993J with an adiabatic shock at $k T_{\text {rev }}=2.1 \mathrm{keV}$ (long dashed) and a radiative shock at $k T_{\text {rev }}=$ $1.0 \mathrm{keV}$ (short dashed), for a $\mathrm{He} / \mathrm{N}$-dominated composition from the $\mathrm{s} 15 \mathrm{~s} 7 \mathrm{~b} 2 \mathrm{f}$ model. Note the strong improvement in the fit to the N VII peak at $0.5 \mathrm{keV}$ compared to Figs. 3-5.
Fe emission at $0.9 \mathrm{keV}$. An example, with two radiative shocks with $T_{1}=1.4 \mathrm{keV}$ and $T_{2}=0.7 \mathrm{keV}$, is shown in Fig. 5 .

In summary, this composition gives an acceptable fit to the observations.

\subsection{The helium/nitrogen zone}

This zone, dominated by $\mathrm{CNO}$ burning products, with He dominant and $\mathrm{N}$ and $\mathrm{Ne}$ the most abundant metals reproduces the emission around $0.5 \mathrm{keV}$ well. The $\mathrm{Fe} \mathrm{L}$ and $\mathrm{NeX}$ peak at $0.9 \mathrm{keV}$ is, on the other hand, overestimated, and there is an obvious lack of emission at $0.6-0.7 \mathrm{keV}$, due to a too low abundance of $\mathrm{O}$. The s15s7b2f model produces an $\mathrm{Fe} \mathrm{L} / \mathrm{NeX}$ peak at slightly lower energy than the $4 \mathrm{H} 47$ model, due to the higher $\mathrm{Ne}$ X-emission in the $4 \mathrm{H} 47$ model. Several emission lines are evident above $1 \mathrm{keV}$, stronger in the s15s7b2f model.
As with the previous models, adding a second shock improves the fit, and the best fit is found for an adiabatic shock at $k T_{\text {rev }}=2.1 \mathrm{keV}$ and a radiative component at $k T_{\text {rev }}=1.0 \mathrm{keV}$ (Fig. 6). This gives the best fit to the spectrum of all the compositions.

The highest temperature component is consistent with an ejecta density gradient of $\eta \sim 12$ if $\tilde{A_{*}}=4$ and $V_{4}=1$ (Table 2).

\subsection{The carbon/oxygen zone}

A C/O-dominated composition gives spectra that do not in any way resemble the observed spectrum. For illustration we show one in Fig. 7. Both the $\mathrm{N}$ peak at $0.5 \mathrm{keV}$ Fe peak at $0.9 \mathrm{keV}$ are completely missing, while the oxygen emission at $0.6-0.7 \mathrm{keV}$ and the $\mathrm{Mg}-\mathrm{S}$ line emission above $1 \mathrm{keV}$ are very strong and dominate the spectrum. Changing the temperature, or adding 


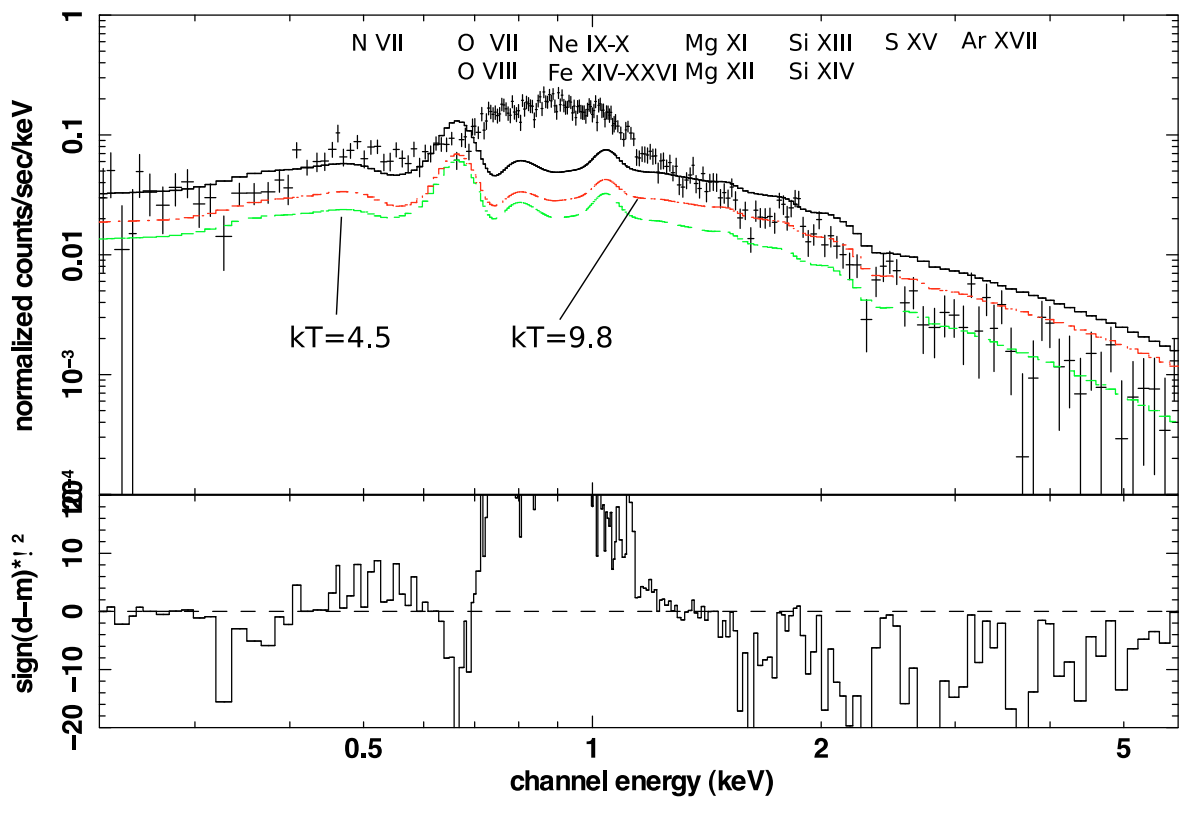

Fig. 7. Fit to the XMM spectrum of SN 1993J with two radiative shocks with $k T_{\text {rev }}=4.5 \mathrm{keV}$ (lower dashed curve) and $k T_{\text {rev }}=9.8$ (upper dashed curve), for a C/O-dominated composition from the $4 \mathrm{H} 47$ model. Note the poor fit at $0.7-1.2 \mathrm{keV}$, ruling out this model. more shocks, modifies the strength of the peaks, but does not result in an overall improvement.

\section{Discussion}

In this paper we have investigated the X-ray spectra of SN 1993J. By applying a hydrodynamical model for a cooling shock to the $\mathrm{X}$-ray observations we have discussed the fits obtained by various compositions taken from realistic ejecta models.

We find that in the spectra of SN 1993J roughly half the contribution comes from an adiabatic shock propagating into a CNO-enriched ejecta, with the rest of the contributions from slower, radiative shocks. These could be caused by instabilities at the reverse shock front or by a clumpy CSM modifying the structure of the interaction region. Comparing ejecta models, we find that the model s15s7b2f (Woosley et al. 1994) gives a somewhat better fit to the line features than the model 4H47 (Nomoto \& Hashimoto 1988; Shigeyama et al. 1994), but the difference is hardly significant. The best fits are obtained by a composition resembling that of the circumstellar ring of SN 1987A. There is no need for a contribution from the circumstellar shock, as was also expected from the low luminosity estimated in Sect. 6.

The observations discussed in the present work are close to the limit of what can be used for spectroscopic studies. If the capabilities of Chandra and XMM had been present at the early phases of this supernova, a much more detailed analysis could have been performed of the X-ray spectra. The next X-ray bright supernova within $\sim 10 \mathrm{Mpc}$ will allow a much more detailed spectroscopic studies of the early spectra. With future missions, like ESA's, NASA's and JAXA's proposed International X-ray Observatory (IXO), these capabilities will be vastly improved. This will allow high $\mathrm{S} / \mathrm{N}$ spectra to be obtained of $\mathrm{SNe}$ at much longer distances, and will also allow detailed spectroscopic studies to be performed also at late times. This will in turn lead to a much more reliable determination of the abundances and temperature in the interaction region. We hope that the analysis in this paper will give some guidance to future X-ray observations of $\mathrm{SNe}$.

Acknowledgements. We are grateful to Stefan Larsson and Linnea Hjalmarsdotter for assistance with XSPEC and to Ken Nomoto and Stan
Woosley for detailed abundance models of SN 1993J. This work has in part been supported by the Swedish National Space Board and the Swedish Research Council. P.C. is a Jansky fellow at the National Radio Astronomy Observatory, which is a facility of the National Science Foundation operated under cooperative agreement by Associated Universities, Inc.

\section{References}

Arnaud, K. A. 1996, in Astronomical Data Analysis Software and Systems V, ed. G. H. Jacoby, \& J. Barnes, ASP Conf. Ser., 101, 17

Arnaud, M., \& Rothenflug, R. 1985, A\&AS, 60, 425

Arnaud, M., \& Raymond, J. 1992, ApJ, 398, 394

Badenes, C., Hughes, J. P., Cassam-Chenaï, G., \& Bravo, E. 2008, ApJ, 680, 11149

Bartel, N., Bietenholz, M. F., Rupen, M. P., et al. 2002, ApJ, 581, 404

Bartel, N., Bietenholz, M. F., Rupen, M. P., \& Dwarkadas, V. V. 2007, ApJ, 668, 924

Chandra, P., Ray, A., \& Bhatnagar, S. 2004, ApJ, 612, 974

Chandra, P., Ray, A., Schlegel, E. M., Sutaria, F. K., \& Pietsch, W. 2005, ApJ, 629,933

Chevalier, R. 1982a, ApJ, 258, 790

Chevalier, R. 1982b, ApJ, 259, 302

Chevalier, R., \& Fransson, C. 1994, ApJ, 420, 268

Decourchelle, A., Ellison, D. C., \& Ballet, J. 2000, ApJ, 543, L57

Dere, K. P., Landi, E., Mason, H. E., Monsignori Fossi, B. C., \& Young, P. R. 1997, A\&AS, 125, 149

Dickey, J. M., \& Lockman, F. J. 1990, ARA\&A, 28, 215

Dwarkadas, V. V., Mioduszewski, A. J., \& Ball, L. T. 2005, in Cosmic Explosions, ed. J. M. Marcaide, \& K. W. Weiler (Springer), IAU Colloq., 192, 47

Ellison, D. C., Decourchelle, A., \& Ballet, J. 2004, A\&A, 413, 189

Filippenko, A. V., Matheson, T., \& Ho, L. C. 1993, ApJ, 415, L103

Fransson, C., \& Sonneborn, G. 1994, in Frontiers of Space and Ground-Based Astronomy, ed. W. Wamsteker, M. S. Longair, \& Y. Kondo (Dordrecht: Kluwer), 249

Fransson, C., Lundqvist, P., \& Chevalier, R. 1996, ApJ, 461, 993

Fransson, C., Challis, P. M., Chevalier, R. A., et al. 2005, ApJ, 622, 991

Ghavamian, P., Laming, J. M., \& Rakowski, C. E. 2007, ApJ, 654, L69

Gröningsson, P., Fransson, C., Lundqvist, P., et al. 2006, A\&A, 456, 581

Houck, J. C., \& Fransson, C. 1996, ApJ, 456, 811

Immler, S., \& Lewin, W. H. G. 2003, in Supernovae and Gamma-Ray Bursters, Lecture notes in physics, ed. K. W. Weiler (Springer), 598, 91

Iwamoto, K., Young, T. R., Nakasato, N., Shigeyama, T., \& Nomoto, K. 1997, ApJ, 477, 865

Kifonidis, K., Plewa, T., Scheck, L., Janka, H.-T., \& Müller, E. 2006, A\&A, 453, 661

Leising, M. D., Kurfess, J. D., Clayton, D. D., et al. 1994, ApJ, 431, L95 
Lundqvist, P., \& Fransson, C. 1996, A\&A, 464, 924

Matzner, C. D., \& McKee, C. F. 1999, ApJ, 510, 379

Maund, J. R., Smartt, S. J., Kudritzki, R. P., Podsiadlowski, P., \& Gilmore, G. F. 2004, Nature, 427, 129

Nahar, S. 1996, ApJS, 106, 213

Nahar, S. 1999, ApJS, 120, 131

Nahar, S., \& Pradhan, A. 1997, ApJS, 111, 339

Nomoto, K., \& Hashimoto, M. 1988, Phys. Rep., 163, 13

Nomoto, K., Suzuki, T., Shigeyama, T., et al. 1993, Nature, 364, 507

Nymark, T. K., Fransson, C., \& Kozma, C. 2006, A\&A, 449, 171

Podsiadlowski, P., Hsu, J. J. L., Joss, P. C., \& Ross, R. R. 1993, Nature, 364, 509

Rakowski, C. E., Badenes, C., Gaensler, B. M., et al. 2006, ApJ, 646, 982

Ray, A., Singh, K. P., \& Sutaria, F. K. 1993, JA\&A, 14, 53

Ripero, J., \& Garcia, F. 1993, IAU Circ., 5731

Shigeyama, T., Suzuki, T., Kumagai, S., et al. 1994, ApJ, 420, 341

Sramek, R. A., \& Weiler, K. W. 2003, in Lecture Notes in Physics, ed. K. W. Weiler (Springer), 598, 145

Swartz, D. A., Ghosh, K. K., McCollough, M. L., et al. 2003, ApJS, 144, 213
Uno, S., Mitsuda, K., Inoue, H., et al. 2002, ApJ, 565, 419

Van Dyk, S. D., Weiler, K. W., Sramek, R. A., Rupen, M. P., \& Panagia, N. 1994, ApJ, 432, L115

Vink, J., Laming, J. M., Gu, M. F., Rasmussen, A., \& Kaastra, J. A. 2003, ApJ, 587, L31

Weiler, K. W., Williams, C. L., Panagia, N., et al. 2007, ApJ, 671, 1959

Woosley, S., Eastman, R. G., Weaver, T. A., \& Pinto, P. 1994, ApJ, 429, 300

Young, P. R., Del Zanna, G., Landi, E., et al. 2003, ApJS, 144, 135

Zatsarinny, O., Gorczyca, T. W., Korista, K. T., Badnell, N. R., \& Savin, D. W. 2003, A\&A, 412, 587

Zatsarinny, O., Gorczyca, T. W., Korista, K. T., Badnell, N. R., \& Savin, D. W. 2004a, A\&A, 417, 1173

Zatsarinny, O., Gorczyca, T. W., Korista, K. T., Badnell, N. R., \& Savin, D. W. 2004b, A\&A, 426, 699

Zhekov, S. A., McCray, R., Borkowski, K. J., Burrows, D. N., \& Park, S. 2006, ApJ, 645, 293

Zimmermann, H.-U., \& Aschenbach, B. 2003, A\&A, 406, 969

Zimmerman, H. U., Lewin, W., Predehl, P., et al. 1994, Nature, 367, 621 